\title{
JULIETTE EPELE
}

\author{
Laboratorio para el Estudio de la Experiencia Musical (LEEM) \\ Facultad de Bellas Artes (FBA) - Universidad Nacional de La Plata (UNLP) \\ epelejuliette@gmail.com
}

Artículo de investigación

\section{Movimiento corporal expresivo en la ejecución solista del piano Indicaciones de carácter e interpretación}

\section{Resumen}

Este trabajo ofrece un estudio sobre percepción musical focalizado en la visualización y caracterización del movimiento corporal de un ejecutante de piano solo, mediante opciones de indicación de carácter tomadas de la partitura interpretada por el pianista. El estímulo consiste en distintos cortes de video de una misma interpretación presentados con y sin sonido, los que fueron apreciados por dos grupos de participantes (músicos pianistas y nomúsicos). Los resultados revelan similitudes en la interpretación corporeizada de los videos, independientemente de la condición, como también diferencias entre los grupos frente al audio, y el audio y video. Esto da cuenta de que el significado musical expresivo está presente tanto en lo visual como en lo sonoro. Asimismo, los datos sustentan la evidencia de que la información visual es un indicador efectivo para la interpretación y el reconocimiento de las diferentes calidades expresivas en base a representaciones compartidas; del mismo modo que, la experiencia musical favorece el desarrollo y acceso a las claves distales de la ejecución, contenida en las formas sónicas en movimiento.

\section{Palabras Clave:}

intención expresiva, interpretación corporeizada, claves distales. 


\title{
JULIETTE EPELE
}

Laboratorio para el Estudio de la Experiencia Musical (LEEM)

Facultad de Bellas Artes (FBA) - Universidad Nacional de La Plata (UNLP)

epelejuliette@gmail.com

Research paper

\section{Embodied expressive movement in solo piano performance \\ Character indicators and interpretation}

\begin{abstract}
This paper shows a study about musical perception. It focuses in the visualization and characterization of a solo piano performer`s body movements, as interpreted in performance according to the character indicators written in the score. The stimulus consisted on different videoclips of the same interpretation that were presented with and without sound to two groups of participants (musicians and non musicians). Results reveal similarities in the embodied interpretation that were not dependent on the condition. On the other hand differences between groups were found according to solo audio and/or audiovisual condition. These results account for a expressive musical meaning that is embedded both in the visual and/or sonic domains. Moreover, data support the evidence that visual information is an efficient indicator for the interpretation and recognition of the different expressive qualities, on the basis of shared representations. Similarly, that musical experience prompts the development and the access to the distal cues of musical performance that are embedded in the sonic forms in movement.
\end{abstract}

\section{Key Words:}

expressive intentionality, embodied interpretation, distal cues. 


\section{Fundamentacion}

"Un intérprete ha de ser capaz. de oír la última nota de su pieza en su oído interno antes de tocar la primera; para conseguirlo, debe crear su propia realización física de la partitura - un término que prefiero al muy manido de la interpretación - de manera más estratégica que táctica, actuando más que reaccionando.” (Barenboim 2007, p. 64).

Todo músico entiende que para decir musicalmente una determinada cosa debe moverse en función de ello. Lo que da por hecho un conocimiento acerca del movimiento específico, y del resultado o efecto sonoro que tal o cual movimiento tiene. Digo específico, en la medida que es propio de una determinada forma de realización vocal o instrumental. De manera tal que, la ejecución musical resulta de una construcción sonora y de movimiento corporal, en la que los elementos expresivos interactúan con los perfiles de producción de los diferentes programas de acción motora.

Según un enfoque corporeizado de la cognición musical los significados expresivos se revelan inseparables de los procesos de producción, sugiriendo un reclutamiento del sistema motor o de la competencia motora en la percepción (Reybrouck 2005; Leman 2008). El cuerpo no sólo hace posible la acción que produce, sino que al mismo tiempo moldea la expresión y es moldeado a través de las sensaciones y de las emociones que experimenta en los permanentes ajustes corporales que tienen lugar en el acto de percepción multisensorial (auditiva, cinética propio- y exteroceptiva, entre otras). De manera que, acción y percepción conforman dos sistemas indisociables, en tanto mutuamente informativos y determinantes para la construcción y el desarrollo de nuestras representaciones y modelos mentales. La integración es clara: en términos funcionales, el sistema corporal requiere y se retroalimenta de información multisensorial y motora, a fin de poder desarrollar y monitorear dinámicamente su desempeño. De ahí que, la realización de nuestras intenciones expresivas resulte de la experiencia interactiva sensorio-motora con el ambiente físico, y con los otros.

Subsiguientemente, la noción de intencionalidad asume una nueva perspectiva a la luz de los aportes de la neurociencia (Iacoboni et al. 2005; Fogassi et al. 2005). En razón de lo cual, nuestro entendimiento del mundo y la atribución de significado remiten a una intencionalidad corporeizada que orienta y organiza nuestra interacción con el ambiente físico. El dispositivo descripto entiende que el reconocimiento de las acciones ejecutadas y la interpretación de la razón por la cual se las ejecuta, son fenómenos relacionados, respaldados - en gran medida - por el mismo mecanismo funcional neuronal. Lo que ofrece una alternativa al estudio del movimiento corporal, por cuanto la comprensión de la intención materializada en la transformación de la energía física se nos revela tanto a través de las particularidades expresivas del discurrir musical - sonido - como de las acciones y de los gestos del ejecutante. 
Los hallazgos sugieren que el conocimiento del significado intencional de la acción es intrínseco al proceso perceptivo/ejecutado y no derivado de este, consecuencia de la evidencia holística que cada acto motor refleja en la secuencia de acciones y de situaciones particulares por él implicadas. Pero, ¿cómo podría este sistema integrado de representación corporeizada participar en la experiencia vívida de la música?

Nuestra participación en la música y la posibilidad de involucrarnos con ella también supone atribución de intencionalidad, basada en la percepción de aspectos expresivos concebidos como parte constitutiva del estado que se adscribe. En tal sentido, la energía física de la música - sonido - ofrece las pistas para una síntesis conforme al patrón gestual contenido en las formas sónicas en movimiento (Leman 2008). Experimentamos la música no como sonido puro, sino como evento multimodal integrado dentro de un marco histórico y cultural; en razón de lo que, la música puede considerarse un agente social virtual capaz de evocar en el oyente resonancias corporales potencialmente significativas. La música nos invita a movernos y a transformar la señal del estímulo en acciones relevantes para la interpretación. Por lo que, cuanto más conozcamos el estilo musical, mejor podremos inferir y predecir la acción distal implicada en la gestualidad del sonido. Su limitada referencialidad, sin embargo, no es sino una de las propiedades más poderosas de la expresión gestual, en la medida en que su significado se funda en la constitución física que universalmente todos los cuerpos humanos compartimos.

Correspondientemente, los estudios sobre comunicación en la temprana infancia (Stern 2010) revelaron un tipo particular de experiencia psicológica de lo vital inherente al acto de movimiento. De acuerdo con los resultados, la naturaleza dinámica de los intercambios afectivos se ve gobernada por configuraciones globales de los rasgos de movimiento, tiempo, espacio, fuerza y dirección de la experiencia sensible. Esto ha dado lugar al concepto de forma o expresión dinámica de vitalidad que, si bien se encuentra directamente asociada al contenido de la experiencia (acto, emoción, modalidad, etc.), refiere a la forma dinámica en sí, antes que al evento o suceso. De este modo, las propiedades o Gestalt emergentes son las que, a través del entonamiento afectivo, permiten la experiencia sentida y compartida de nuestros movimientos en la interacción intersubjetiva. Y, en tanto amodales, las formas dinámicas sugieren una conexión a nivel de gestalt común entre los significados de las modalidades expresivas sonora y corporal; diríase, una dimensión de intercambio de la propia experiencia en la que la expresión y la comunicación se hacen vívidas.

En este contexto, los trabajos de Jane Davidson han sido pioneros en explorar las relaciones entre la estructura musical, los movimientos que tienen lugar en la ejecución musical y los significados emergentes; dando cuenta de que los movimientos de los ejecutantes conllevan información que ayuda a clarificar e intensificar el significado expresivo de la interpretación musical. Una de sus primeras investigaciones (Davidson 1993) evidenció que el movimiento corporal puede re- 
velar las intenciones - inexpresiva, expresiva, exagerada - de una interpretación, a la vez que demostró que la información visual es un indicador efectivo para la estimación de las distintas maneras expresivas por parte del espectador. Davidson (1994, 2002) exploró, a su vez, los tipos de movimientos que pueden guiar las percepciones de observadores/oyentes respecto de una ejecución expresiva en el piano, revelando que los movimientos de la región alta del torso/cabeza del ejecutante son suficientes para un juicio del observador respecto de las intenciones expresivas, pero que si se los combina con la observación de los movimientos de las manos, la información es aún más precisa. También estudió los gestos pianísticos utilizados para la expresión musical (Davidson 2007), buscando elaborar un repertorio de movimiento gestual asociado a los modos particulares o a las indicaciones dadas por el autor al ejecutante. Sin embargo, los resultados evidenciaron que tal vez sea la calidad del movimiento, y no los movimientos específicos en sí, la que guía la detección de los diferentes modos intencionales expresivos.

Por otra parte, en los últimos años se ha asistido a un renacimiento de la valoración del cuerpo con relación al estudio sobre la experiencia musical. Por lo que, la observación del cuerpo en movimiento y del modo en que éste se desarrolla y adquiere significado en la performance ha devenido en uno de los focos más importantes de la investigación musical, aportando claves hasta el momento no incluidas en los análisis tradicionales sobre ejecución e interpretación. Se ha visto que los movimientos corporales oscilatorios son parte integral de la biomecánica y de la morfología de la dinámica corporal utilizados para dar cuenta de los aspectos tanto temporales como dinámicos de la ejecución musical expresiva (Naveda y Leman 2011). Asimismo, el análisis de patrones de velocidad permitió comprobar que tanto los ejecutantes como los oyentes comparten una sensibilidad para entonar corporalmente con las formas sonoro-kinéticas emergentes de la performance instrumental (Leman et al. 2009). Por otra parte, se encontró que la imaginación ideomotora y los perfiles dinámicos vitales experimentados en intérpretes y oyentes permiten acordar con descriptores verbales de la expresividad musical (Martínez y Pereira Ghiena 2013; Epele y Martínez 2011).

Por lo tanto, este trabajo ofrece una investigación empírica sobre percepción musical focalizada en la visualización y caracterización del movimiento corporal de un ejecutante de piano solo desde el punto de vista de la expresión. El objetivo del trabajo es estudiar en qué medida los movimientos del pianista revelan en la interpretación las intenciones expresivas relativas a las indicaciones de carácter dadas por el autor. Se trata, en verdad, de una doble interpretación: por un lado, la que refiere a la transformación de las energías y realización física - en términos de Barenboim - de las ideas musicales en la ejecución y, por el otro, la que traduce perceptivamente las acciones observables y las asocia a las imágenes y a la experiencia corporal que los diferentes conceptos evocan o significan en el espectador. 


\section{Objetivos}

Es propósito de este estudio obtener evidencia empírica acerca de la comunicación de los aspectos expresivos a través del movimiento corporal en la ejecución pianística, en razón de lo cual se pretende:

- Encontrar posibles correspondencias entre el movimiento corporal en la ejecución solista del piano y la interpretación corporeizada que los receptores hagan a partir de términos específicos, indicadores del carácter musical.

- Descubrir si tanto la imagen con sonido como la imagen sola de los movimientos realizados por el ejecutante o el sonido solo revelan al espectador el carácter de su interpretación musical.

- Detectar si la experiencia musical favorece la inferencia de indicios distales que den lugar a la interpretación del carácter a partir de las señales proporcionadas por el estímulo.

\section{Metodología}

\section{Participantes}

Participaron de este experimento 46 jóvenes y adultos, de los cuales: i) 22 son músicos egresados de la carrera de Instrumento-Piano de diferentes institutos de formación académica clásica de la Provincia de Buenos Aires, algunos de ellos músicos profesionales ( 7 varones y 16 mujeres, entre 32 y 60 años de edad, media $=45.36$ ) y ii) 22 no-músicos con baja o nula familiaridad con la música académica ( 9 varones y 14 mujeres, entre 26 y 69 años de edad, media $=39.65), 4$ de los cuales tomaron clases particulares de instrumento - no piano - durante 5 años o menos, y otros 6, en cambio, durante siquiera 1 año.

\section{Estímulo}

Consistió en diez cortes de video de una misma interpretación a cargo del pianista Daniel Barenboim de la obra: Tras una lectura de Dante. Fantasia quasi Sonata de Franz Liszt, presentados en orden diferente a cada participante, según las modalidades: i) auditiva, ii) visual, y iii) audiovisual; lo que hizo un total de 30 cortes. La selección de los mismos remite a distintos momentos expresivos de la obra según se indica en la partitura, y su duración varia a fin de preservar la unidad de significado musical de cada fragmento (compases 35-47; 54-66; 103-115; 115-123; 124-135; 157-166; 179-184; 222-236; 276-292; 369-376). Los cortes representan ejemplos musicales contrapuestos en rango dinámico "ffff?" (ejemplos 02, 03, 04, 07, 08, 10) o "p-ppp" (01, 05, 06, 09), tempo rápido $(01,02,03,07,08)$ o lento $(04$, 
05, 06, 09, 10), y motivos temáticos desarrollados: introductorio (c.1) (ejemplos 04, 08, 10), tema A (c.35) (ejemplos 01, 02, 05, 06, 09) o tema B (c.103) (ejemplos 03, 07). Dos de los cortes poseen igual indicación de carácter (ejemplos 01 y 09), en tanto que un tercer fragmento carece de indicación escrita (ejemplo 08), a fin de poder contrastar los resultados. En cuanto a las tomas, éstas permitieron la observación del teclado y del pianista desde la banqueta hacia arriba, siempre desde un mismo lado.

Respecto de la obra: Tras una lectura de Dante. Fantasia quasi Sonata, como se la conoce desde 1858, muy probablemente en alusión al poema homónimo de Victor Hugo contenido en Las Voces Interiores, no se tienen detalles de una composición más allá de la música pura, aun considerando los títulos inicialmente sugeridos por el autor para la versión de 1849: Paralipómenos o Prolegómenos a la Divina Comedia, y la ilustración del autor que acompañó la primera edición y que representaba a una mujer meditando (una musa?) con un arpa. Más próxima a una fantasía que a una sonata desde el punto de vista formal, la obra se compone de un solo movimiento en el que se entrelazan los elementos motívicos siguiendo la técnica de la transformación temática, según la cual éstos alcanzan estados de condensada vehemencia para devenir, poco después, en melodías de apacible serenidad y dulzura. La unidad orgánica que se desprende de la metamorfosis de los temas, así como su extraordinaria intensidad dramática, convierten a la pieza en una obra de gran envergadura y virtuosismo pianístico. Por lo demás, las indicaciones en partitura son todas originales y representativas del estilo de uno de los compositores del romanticismo musical por excelencia.

Los diez cortes de video realizados para este trabajo se explicitan a continuación mediante sus marcas de tiempo inicial y final respecto del video original, los compases a que corresponde el fragmento musical pertinente, y el carácter indicado por el compositor en cada uno de los pasajes: Video 01: 1.19.26 - 1.20.00 (c. 35-47) "lamentoso"; Video 02: 1.20.13 - 1.20.38 (c.54-66) "disperato"; Video 03: 1.21 .45 - 1.22.13 (c.103-115) "precipitato"; Video 04: 1.22 .13 - 1.22.40 (C.115123) "maestoso"; Video 05: 1.22 .46 - 1.23.43 (c.124-135) "dolcissimo con intimo sentimento"; Video 06: 1.25.16 - 1.26 .07 (c.157-166) "dolcissimo con amore"; Video 07: 1.26 .36 - 1.26 .54 (c.179-184) "appassionato assai"; Video 08: 1.22 .10 1.28.36 (222-236); Video 09: 1.29.58 - 1.30.29 (c.276-292) "lamentoso"; Video 10: 1.33 .44 - 1.34.21 (c.369-376) "marcatissimo".

\section{Aparatos}

Los cortes y su procesamiento se realizaron mediante el programa de edición de videos Sony Vegas Pro 10, y el análisis de los datos por medio del programa estadístico SPSS 20.0 para Windows. 


\section{Procedimiento}

Se les pidió a los participantes que vieran los videos, sin especificaciones acerca de la obra, el autor o el estilo musical interpretado, y que estimaran el carácter del movimiento corporal del ejecutante en cada corte, puntuando una lista predeterminada de 8 términos indicadores del carácter musical según una escala de 7 niveles, donde $0=$ nula correspondencia y $6=$ correspondencia absoluta. Los términos que conforman la lista - originalmente escritos en italiano y traducidos al español para el grupo de no-músicos, por cuanto éstos desconocen el vocabulario musical académico convencional - fueron relevados de las secciones de la partitura concernientes a los cortes, por lo que constituyen indicaciones originales del propio Liszt bajo interpretación del maestro Barenboim - dato que sí fue revelado a los participantes. He aquí la lista de los términos: "lamentoso", "dolcissimo con intimo sentimento", "dolcissimo con amore", "appassionato assai", "maestoso", "disperato", "precipitato", "marcatissimo".

\section{Resultados}

Para el estudio de los datos se realizó un análisis de varianza con factores múltiples de medidas repetidas, atendiendo a las variables: video (corte), condición (auditiva, visual y audiovisual), indicación de carácter y tipo (músico o no-músico), el cual arrojó diferencias significativas para los factores: video $(\mathrm{F}=12.898, p<, 000)$ e indicación de carácter $(\mathrm{F}=36,471, p<, 000)$; del mismo modo que para las interacciones entre: video y condición $(\mathrm{F}=3,655, p<, 002)$, condición e indicación de carácter $(\mathrm{F}=5,334, p<, 000)$, y condición y tipo $(\mathrm{F}=5,301, p<, 000)$; en tanto que, las diferencias en la interacción entre indicación de carácter y tipo resultaron marginalmente significativas $(\mathrm{F}=2,160, p<, 062)$.

De los resultados anteriores, se destaca la relevancia de la condición o modalidad de presentación de los cortes de video en la interpretación de cada uno de los grupos de participantes, sean éstos músicos o no-músicos; lo que habla de diferencias en la percepción de la condición según el tipo. Esto puede apreciarse también en el gráfico de la figura 1, el cual representa las particularidades de tal interacción evidenciando similitudes entre los grupos en la percepción de la condición visual, y aún audiovisual en contraste con la condición de presentación del audio solo; así como que los resultados promedio para los músicos son similares en las tres condiciones; en tanto que, para los no-músicos existe una marcada diferencia en la condición del audio solo, al punto de ser ésta estadísticamente distinta de la respuesta promedio brindada por los músicos, por cuanto las medias estimadas para los grupos a través de un intervalo de confianza del $95 \%$, no se superponen en sus valores. 


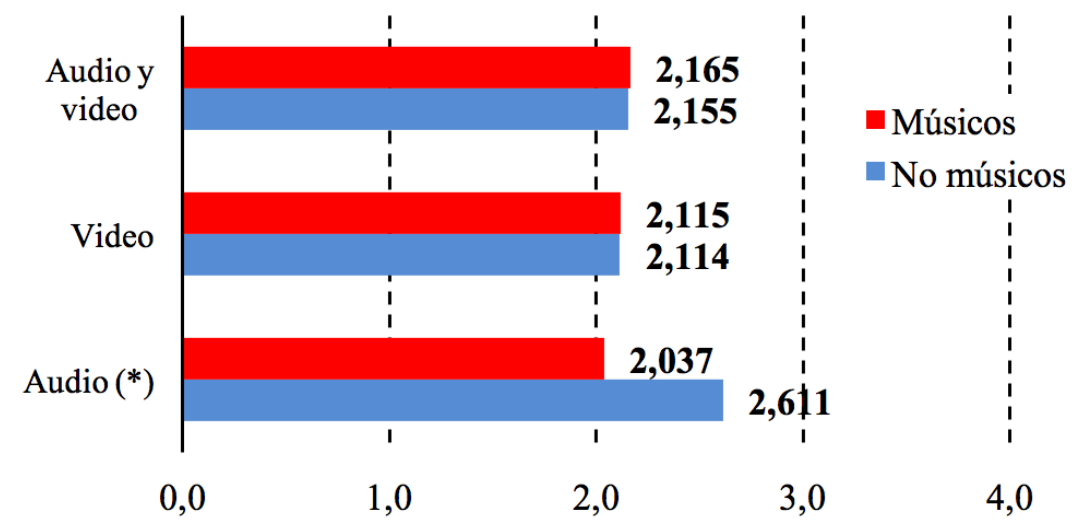

Figura 1. Valoración promedio de las condiciones auditiva, visual y audiovisual para los músicos y los no-músicos, donde $(*)$ señala una diferencia estadísticamente significativa con un nivel de confianza del $95 \%$.

\section{MúsICOS}

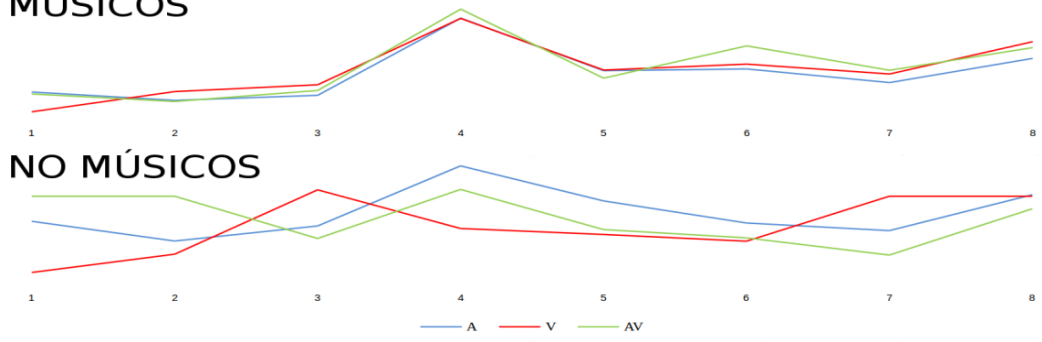

Figura 2. Visualización de las estimaciones promedio realizadas por los músicos y los no-músicos en las condiciones audiovisual, visual y auditiva para las 8 indicaciones de carácter propuestas $(1=$ lamentoso; $2=$ dolcissimo con amore; $3=$ dolcissimo con intimo sentimento; $4=$ appassionato assai; $5=$ maestoso; $6=$ disperato $; 7=$ precipitato $; 8=$ marcatissimo $)$.

En cuanto al uso de los términos, aún cuando la interacción entre las indicaciones de carácter y la condición de presentación de los cortes es marginalmente significativa, se observa mayor homogeneidad en las apreciaciones de los músicos que en las de los no-músicos, a la vez que una leve aproximación entre los resultados concernientes a las condiciones audiovisual y auditiva respecto de los obtenidos bajo la condición solo imagen. Los detalles de tal interacción se aprecian en la figura 2. 


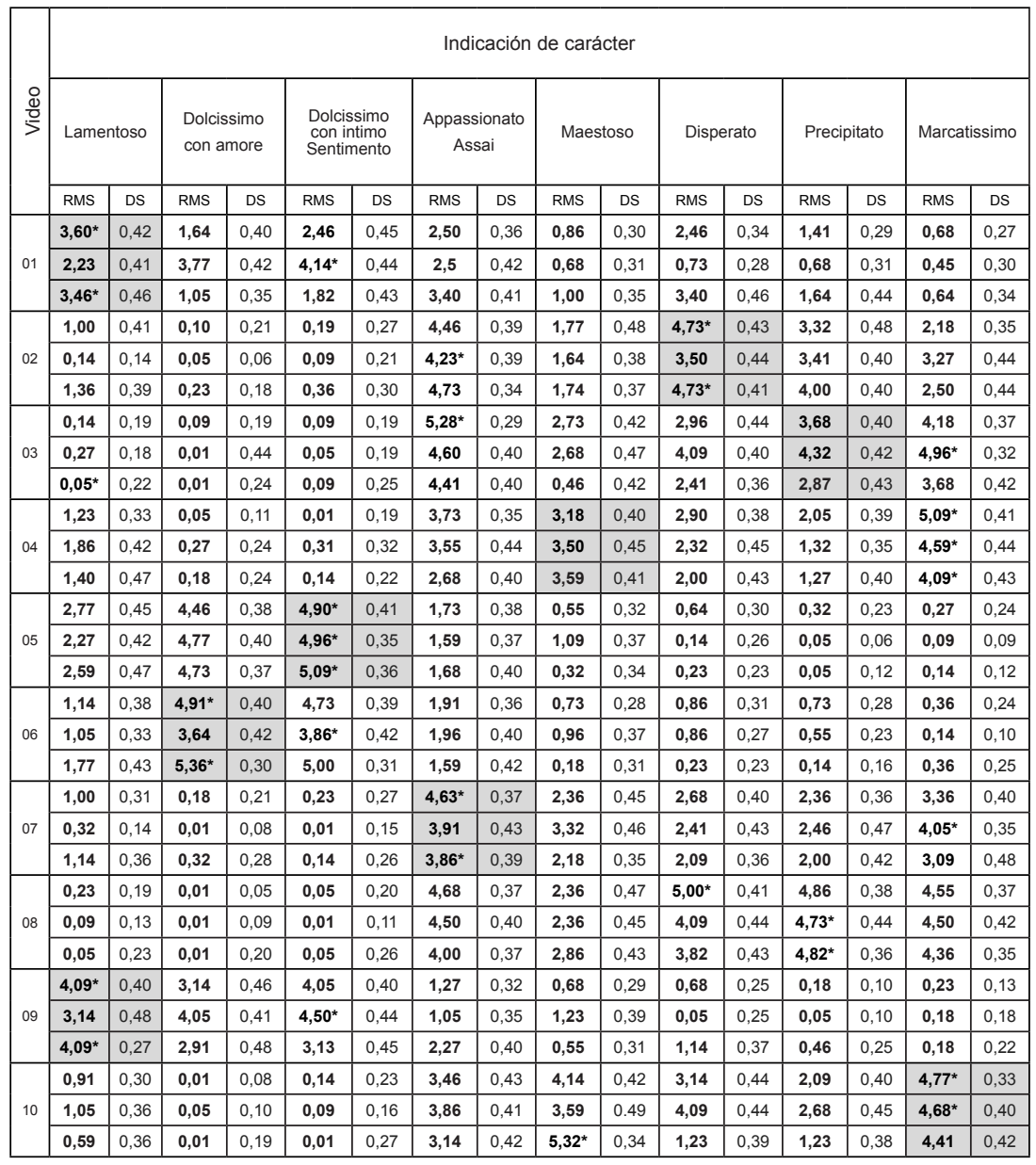

Tabla 1. Valores medios y desviación estándar del puntaje asignado por los músicos a cada indicación de carácter según el video y la condición (de arriba hacia abajo): audiovisual, visual y auditiva. Los casilleros sombreados corresponden al carácter indicado en la partitura. El asterisco señala la opción mayormente puntuada.

A propósito, un análisis pormenorizado de los valores medios obtenidos a partir de las apreciaciones de los participantes con relación a cada uno de los términos propuestos, permite señalar que la indicación de carácter mayormente puntuada por los músicos para caracterizar cada uno de los fragmentos de video coincide con el carácter musical indicado en la partitura en 7 de los 9 cortes presentados bajo las condiciones audiovisual y auditiva (videos: 01, 02, 05, 06, 07, 09 y 10) y sólo en 2 de los cortes en los que la imagen se presenta sin sonido (videos: 
05 y 10); mientras que, en los no-músicos, las coincidencias se dan en apenas 3 de los cortes bajo las modalidades audiovisual y auditiva (videos 05, 09 y 10, y 01, 09 y 10 , respectivamente) y en 2 de los cortes presentados según la modalidad visual (videos: 05 y 10). Recordemos que el video 08 no presenta indicación de carácter. Con lo cual, los resultados revelan mayor distinción y precisión en el uso de las palabras por parte de los participantes, particularmente de los músicos, cuando el sonido se hace presente; así como un acuerdo en la caracterización de los videos 05 y 10, independientemente de la experticia y de la condición; lo que, probablemente, remita a una mayor claridad expresiva e interpretativa de la actuación del pianista, singularmente manifiesta en estos dos ejemplos de video. Las respuestas referidas a los videos 03 y 04, por ejemplo, en los que la opción más puntuada no coincide con la indicación en partitura, muestran, sin embargo, similitudes entre los grupos bajo las condiciones audiovisual y auditiva. En tanto que, las restantes respuestas más puntuadas tendieron a recaer sobre las indicaciones: "dolcissimo con intimo sentimento" o "marcatisssimo", revelando la existencia de opciones más significativas o representativas, así como una estimación polarizada de las mismas en razón de una apreciación contrapuesta o contrastante del carácter expresado (Ver tablas 1 y 2).

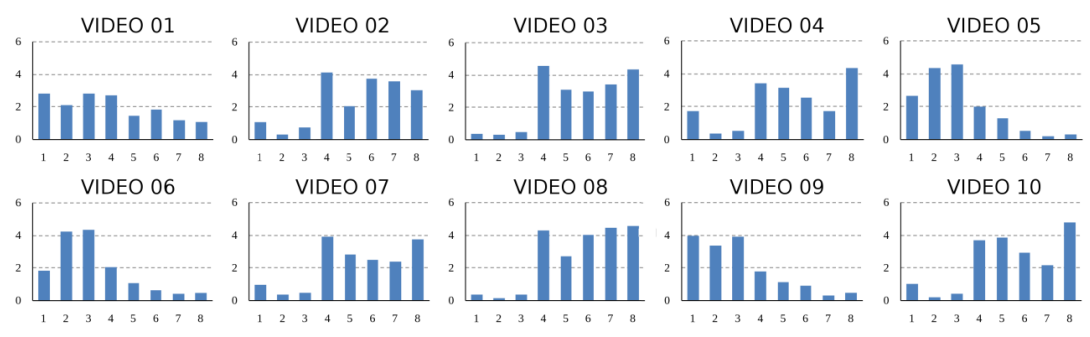

Figura 3. Visualización de las estimaciones promedio realizadas por los participantes para cada uno de los 10 videos. Sobre el eje x se representan las 8 las indicaciones de carácter valoradas $(1=$ lamentoso; $2=$ dolcissimo con amore; $3=$ dolcissimo con intimo sentimento; $4=$ appassionato assai $; 5=$ maestoso; $6=$ disperato $; 7=$ precipitato $; 8=$ marcatissimo) según una escala de 0 a 6 .

Por otra parte, una consideración general e integrada de las valoraciones realizadas sobre las indicaciones de carácter indica mayor determinación en el descarte de las opciones que en la elección de una única alternativa, al tiempo que evidencia una tendencia a la agrupación de los términos, conforme a: "lamentoso", "dolcissimo con amore" y "dolcissimo con intimo sentimento" vs. "disperato", "precipitato" y "marcatissimo", en tanto que "appassionato assai" y "maestoso" resultan más independientes (figura 3). Esto da cuenta de una valoración relativa y contrapuesta del carácter, aparentemente, referida a un mayor o menor monto de energía o intensidad expresiva. De ahí que, según las veces, las adjetivaciones 
funcionen, también, de manera más o menos neutral. Respectivamente, los videos 05, 06 y 09, e inclusive el 01 - menos claro - en los que los términos "maestoso", "disperato", "precipitato" y "marcatissimo" recibieron valoraciones cercanas o inferiores a 1, corresponden a fragmentos musicales con indicación de carácter "lamentoso" o "dolcissimo", e indicación dinámica dentro del rango p-ppp.

\begin{tabular}{|c|c|c|c|c|c|c|c|c|c|c|c|c|c|c|c|c|}
\hline \multirow{3}{*}{$\frac{8}{8}$} & \multicolumn{16}{|c|}{ Indicación de carácter } \\
\hline & \multicolumn{2}{|c|}{ Lamentoso } & \multicolumn{2}{|c|}{$\begin{array}{l}\text { Dolcissimo } \\
\text { con amore }\end{array}$} & \multicolumn{2}{|c|}{$\begin{array}{l}\text { Dolcissimo } \\
\text { con intimo } \\
\text { Sentimento }\end{array}$} & \multicolumn{2}{|c|}{$\begin{array}{c}\text { Appassionato } \\
\text { Assai }\end{array}$} & \multicolumn{2}{|c|}{ Maestoso } & \multicolumn{2}{|c|}{ Disperato } & \multicolumn{2}{|c|}{ Precipitato } & \multicolumn{2}{|c|}{ Marcatissimo } \\
\hline & RMS & DS & RMS & DS & RMS & DS & RMS & DS & RMS & DS & RMS & DS & RMS & DS & RMS & DS \\
\hline \multirow{3}{*}{01} & 2,41 & 0,42 & 2,36 & 0,40 & $2,77^{\star}$ & 0,45 & 2,55 & 0,36 & 1,46 & 0,30 & 1,27 & 0,34 & 0,50 & 0,29 & 0,82 & 0,28 \\
\hline & 1,55 & 0,41 & 2,00 & 0,42 & $2,86^{\star}$ & 0,44 & 2,32 & 0,42 & 1,77 & 0,31 & 0,82 & 0,28 & 0,96 & 0,32 & 1,91 & 0,30 \\
\hline & $3,77^{*}$ & 0,46 & 1,91 & 0,35 & 2,72 & 0,43 & 3,14 & 0,41 & 2,86 & 0,35 & 2,50 & 0,46 & 1,82 & 0,44 & 1,86 & 0,34 \\
\hline \multirow{3}{*}{02} & 1,82 & 0,40 & 0,73 & 0,21 & 1,41 & 0,27 & $3,91^{*}$ & 0,38 & 2,32 & 0,38 & 3,27 & 0,43 & 2,73 & 0,48 & 2,86 & 0,35 \\
\hline & 0,27 & 0,14 & 0,09 & 0,06 & 0,59 & 0,21 & 3,23 & 0,38 & 1,96 & 0,38 & 2,96 & 0,44 & $4,23^{*}$ & 0,40 & 3,32 & 0,44 \\
\hline & 1,86 & 0,39 & 0,77 & 0,18 & 1,73 & 0,30 & $4,36^{*}$ & 0,34 & 3,05 & 0,37 & 3,36 & 0,42 & 3,86 & 0,40 & 4,18 & 0,44 \\
\hline \multirow{3}{*}{03} & 0,59 & 1,19 & 0,55 & 0,19 & 0,73 & 0,19 & $4,50^{*}$ & 0,29 & 3,50 & 0,42 & 2,91 & 0,44 & 3,27 & 0,44 & 4,23 & 0,37 \\
\hline & 0,36 & 0,18 & 0,09 & 0,04 & 0,55 & 0,19 & 4,64 & 0,40 & 2,91 & 0,46 & 3,64 & 0,39 & 3,36 & 0,42 & $4,68^{*}$ & 0,32 \\
\hline & 0,68 & 0,21 & 1,14 & 0,24 & 1,46 & 0,24 & 4,09 & 0,40 & 3,23 & 0,43 & 1,91 & 0,36 & 2,86 & 0,43 & $4,23^{*}$ & 0,42 \\
\hline \multirow{3}{*}{04} & 1,59 & 0,33 & 0,32 & 0,11 & 0,77 & 0,19 & 3,73 & 0,36 & 2,96 & 0,40 & 2,73 & 0,38 & 1,77 & 0,39 & $4,46^{*}$ & 0,41 \\
\hline & 1,18 & 0,41 & 0,46 & 0,24 & 1,05 & 0,32 & 3,50 & 0,44 & 2,59 & 0,45 & 2,46 & 0,45 & 1,86 & 0,35 & $4,09^{\star}$ & 0,44 \\
\hline & 3,09 & 0,47 & 0,82 & 0,24 & 0,96 & 0,22 & 3,18 & 0,39 & 3,00 & 0,41 & 2,73 & 0,43 & 2,23 & 0,41 & $3,68^{*}$ & 0,24 \\
\hline \multirow{3}{*}{05} & 3,00 & 0,45 & 4,00 & 0,38 & $4,05^{\star}$ & 0,41 & 1,91 & 0,38 & 1,50 & 0,32 & 0,64 & 0,30 & 0,27 & 0,23 & 0,55 & 0,24 \\
\hline & 2,38 & 0,41 & 3,32 & 0,40 & $4,05^{*}$ & 0,35 & 2,32 & 0,37 & 2,46 & 0,37 & 0,77 & 0,26 & 0,14 & 0,06 & 0,32 & 0,10 \\
\hline & 2,64 & 0,47 & $4,77^{\star}$ & 0,37 & 4,50 & 0,36 & 2,64 & 0,40 & 1,82 & 0,34 & 0,82 & 0,27 & 0,41 & 0,12 & 0,46 & 0,46 \\
\hline \multirow{3}{*}{06} & 1,86 & 2,15 & 3,55 & 1,41 & $4,27^{*}$ & 1,94 & 1,77 & 1,69 & 1,14 & 1,69 & 0,68 & 1,37 & 0,27 & 0,74 & 0,50 & 0,88 \\
\hline & 2,00 & 0,33 & 2,96 & 0,42 & $3,05^{*}$ & 0,42 & 1,86 & 0,40 & 1,32 & 0,37 & 0,46 & 0,27 & 0,36 & 0,23 & 0,32 & 0,10 \\
\hline & 3,14 & 0,43 & 4,96 & 0,30 & $5,23^{*}$ & 0,31 & 3,14 & 0,41 & 2,00 & 0,31 & 0,86 & 0,23 & 0,59 & 0,16 & 1,05 & 0,25 \\
\hline \multirow{3}{*}{07} & 1,23 & 0,31 & 0,73 & 0,20 & 1,23 & 0,27 & 3,14 & 0,38 & 2,86 & 0,45 & 2,23 & 0,40 & 1,96 & 0,36 & $3,82^{\star}$ & 0,40 \\
\hline & 0,32 & 0,14 & 0,18 & 0,08 & 0,46 & 0,15 & 3,91 & 0,43 & 2,82 & 0,46 & 2,91 & 0,43 & 2,82 & 0,47 & $4,96^{*}$ & 0,35 \\
\hline & 1,64 & 0,36 & 0,91 & 0,28 & 0,96 & 0,26 & $4,05^{*}$ & 0,39 & 3,50 & 0,35 & 2,50 & 0,33 & 2,59 & 0,42 & 3,36 & 0,48 \\
\hline \multirow{3}{*}{08} & 0,50 & 0,19 & 0,14 & 0,05 & 0,64 & 0,20 & 4,32 & 0,37 & 2,68 & 0,47 & 3,64 & 0,41 & 3,82 & 0,39 & $4,36^{*}$ & 0,37 \\
\hline & 0,41 & 0,13 & 0,18 & 0,09 & 0,23 & 0,11 & 4,14 & 0,40 & 2,59 & 0,45 & 3,68 & 0,44 & 4,00 & 0,43 & $4,77^{*}$ & 0,42 \\
\hline & 1,05 & 0,23 & 0,73 & 0,20 & 1,09 & 0,26 & 4,00 & 0,38 & 3,36 & 0,43 & 3,73 & 0,43 & 4,50 & 0,36 & $4,96^{*}$ & 0,36 \\
\hline \multirow{3}{*}{09} & $4,22^{\star}$ & 0,40 & 2,64 & 0,46 & 4,00 & 0,40 & 1,36 & 0,32 & 1,09 & 0,29 & 0,59 & 0,25 & 0,23 & 0,10 & 0,41 & 0,13 \\
\hline & 3,23 & 0,48 & 3,91 & 0,41 & $3.96^{\star}$ & 0,44 & 1,64 & 0,35 & 1,32 & 0,39 & 0,82 & 0,25 & 0,23 & 0,10 & 0,46 & 0,19 \\
\hline & $5,00^{*}$ & 0,28 & 3,41 & 0,48 & 3,68 & 0,45 & 3,27 & 0,40 & 1,86 & 0,31 & 2,14 & 0,37 & 0,82 & 0,25 & 1,27 & 0,22 \\
\hline \multirow{3}{*}{10} & 0,77 & 0,30 & 0,18 & 0,08 & 0,77 & 0,22 & 3,46 & 0,43 & 3,05 & 0,42 & 2,82 & 0,44 & 2,18 & 0,40 & $5,00^{*}$ & 0,32 \\
\hline & 1,36 & 0,36 & 0,23 & 0,10 & 0,50 & 0,17 & 3,50 & 0,41 & 3,05 & 0,49 & 3,00 & 0,44 & 2,09 & 0,45 & $4,86^{*}$ & 0,41 \\
\hline & 1,46 & 0,36 & 0,69 & 0,19 & 1,00 & 0,27 & 4,32 & 0,42 & 4,00 & 0,34 & 3,41 & 0,39 & 1,23 & 0,37 & $4,96^{*}$ & 0,42 \\
\hline
\end{tabular}

Tabla 2. Valores medios y desviación estándar del puntaje asignado por los no-músicos a cada indicación de carácter según el video y la condición (de arriba hacia abajo): audiovisual, visual y auditiva. Los casilleros sombreados corresponden al carácter indicado en la partitura. El asterisco señala la opción mayormente puntuada. 


\section{Discusión}

El propósito del presente trabajo ha sido estudiar, a través de la experiencia perceptiva de espectadores músicos (pianistas) y no-músicos, el conocimiento y la comprensión de la intención expresiva en la ejecución de piano solo, relativa al carácter de la interpretación musical. Para ello, se diseñó un trabajo focalizado en la audición y visualización de una interpretación pianística bajo tres condiciones (sonido-imagen, imagen sola y sonido solo), a fin de obtener evidencia empírica a favor de la hipótesis de que el significado intencional se nos revela consustancial a los procesos y a los modos (sonoro / kinético) de producción implicados en la realización del evento. Se utilizó como herramienta de testeo una lista de 8 indicaciones de carácter tomadas de la partitura, puntualmente, de los fragmentos correspondientes a los cortes, abriendo a su vez juego a la interpretación de las palabras cuyo elemento más importante es, en este tipo de indicaciones, una calidad y, en consecuencia, su valor de uso. Por otra parte, se consideró el potencial efecto que el conocimiento musical experto podía tener en la experiencia del participante durante la tarea. Los resultados muestran que las percepciones de los participantes son similares frente a las condiciones visual y audiovisual. Lo que de ningún modo implica un comportamiento idéntico entre los grupos de participantes.

En efecto, músicos y no-músicos configuraron de manera diferente su apreciación de los aspectos expresivos, tal como lo revelan las distintas valoraciones de los términos realizadas por ambos grupo (figura 2). Respecto de lo que, los primeros mostraron un ajuste y mayor precisión en la caracterización de los estímulos audiovisual y auditivo, referido al hecho de que sus descripciones de los videos y condiciones, remitidas a la opción de carácter más puntuada, recurrieron al uso de una mayor variedad de términos, reflejando la indicación en partitura en 7 de 9 videos (recordemos que el video 08 no presenta indicación escrita) contra 3 respuestas coincidentes en relación a la caracterización de los no-músicos. Una posible explicación podría vincularse al hecho de que el sonido ofrece al experto una información que permite no ya una resonancia corporal intencionada a partir de las claves proximales emergentes, sino la comprensión de la técnica específica de movimiento corporal funcional al estilo musical, sustentando la hipótesis de que el conocimiento especializado favorece la inferencia de claves distales relativas a la ejecución e interpretación de la intención expresiva en la música. Siendo así, este conocimiento permitió, también, a los pianistas interpretar con exhaustividad las implicancias técnico-expresivas relativas a las indicaciones de carácter propuestas, características y propias del estilo musical romántico de Liszt.

En los restantes casos, en cambio, las repuestas más puntuadas tendieron a polarizar las opciones entre las más representativas de un carácter más o menos enérgico, limitando la caracterización de los videos al uso de, preponderantemente, dos indicaciones: "marcatissimo" y "dolcissimo con intimo sentimento". Esto 
podría deberse al hecho de que la herramienta de testeo aludida - indicaciones de carácter, o adjetivos - si bien sugiere una intención que puede ser descripta, opera, en realidad, calificando, o des-objetivando una idea de manera relativa. A propósito, algunos de los participantes manifestaron hallarse frente a la complejidad de repensar el significado de las indicaciones en función de lo que veían y/o escuchaban en los videos, pudiendo establecer distintas relaciones de proporción o asociaciones entre éstas. De ahí que, las opciones de carácter funcionasen a manera de grupos contrapuestos - algunas veces, más neutrales - conforme al monto de energía implicado. Dicha no univocidad entre la palabra y la música, sin embargo, no invalida la experiencia corporeizada de los espectadores quienes, por otra parte, valoraron de manera diferenciada las intenciones expresivas del ejecutante en cada corte según lo refirieron en la caracterización de los mismos.

Puntualmente, el fragmento musical correspondiente al video 08, sin indicación en partitura, arrojó resultados concordantes a los obtenidos en las restantes descripciones respecto de la estimación de las condiciones y la valoración de las indicaciones de carácter por parte de los grupos. Esto evidencia, por un lado, el modo particular de operar de las adjetivaciones antes descripto, como también la cuestión de que el carácter de la interpretación pianística, más allá de las palabras, se trasluce tanto en lo visual como en lo sonoro, brindando, en cualquier caso, las pistas para la decodificación de los significados y de la intencionalidad expresiva, contenidos en las formas de movimiento sónico y corporal.

Por otra parte, la descripción en términos coincidentes con que los músicos caracterizaron los videos 01 y 09 - ambos con indicación "lamentoso" - dio cuenta de la apreciación de aspectos expresivos comunes a estos dos cortes; pese a las diferencias entre los fragmentos que, también, quedaron reflejadas en la estimación del total de las opciones elaborada por los participantes para cada video. Esto favorece, una vez más, la noción de que el saber experto facilita el acceso a las claves distales, al mismo tiempo que sugiere la existencia de indicadores dinámicos globales de la calidad, asociados al significado de la expresión musical. El dato se ciñe, en principio, exclusivamente, a la respuesta dada por los pianistas frente a las condiciones auditiva y audiovisual. No obstante, las observaciones anteriores sustentarían igualmente esta premisa.

Para terminar, si bien los resultados referidos a la adjetivación muestran similitudes en la apreciación de las condiciones auditiva y audiovisual por parte de los músicos, evidenciando el peso del conocimiento experto en la interpretación de las indicaciones de carácter con relación al estilo de ejecución musical; la caracterización de los videos considerada en términos generales, sin embargo, revela tendencias comunes entre las condiciones visual y audiovisual para parte de los dos grupos, reflejada tanto en el modo en que los participantes asociaron y contrapusieron las palabras, como en lo relativo a la interpretación de las condiciones de presentación de los videos. De este modo, la percepción del carácter musical y de su realización física dio cuenta de aspectos expresivos comunes entre las 
modalidades expresivas sonora y corporal, por cuanto la sola visualización de los gestos del ejecutante permitió, igualmente, al espectador descubrir el carácter de la interpretación musical en el piano.

\section{Referencias}

Barenboim, D. (2007). El Sonido es Vida: El Poder de la Música. Bogotá: Grupo Editorial Norma.

Davidson, J. W. (1993). Visual perception of manner in the movements of solo musicians. Psychology of Music, 21, 103-113.

Davidson, J. W. (1994). What type of information is conveyed in the body movements of solo musician performers? Journal of Human Movement Studies, 6, 279-301.

Davidson, J. W. (2002). Understanding the expressive movements of a piano solo. Musikpsychologie 16, 9-13.

Davidson, J. W. (2007). Qualitative insights into the use of expressive body movements in solo piano performance: a case study approach. Psychology of Music, 35, 3, 381-401.

Epele, J. y Martínez I. C. (2011). Parámetros de articulación y simulación ideomotora. En A. Pereira Ghiena, P. Jacquier, M. Valles y M. Martínez (Eds.). Musicalidad Humana. Actas de la X Reunión Anual de la Sociedad Argentina para las Ciencias Cognitivas de la Música. Buenos Aires. SACCoM, pp. 509-520.

Fogassi, L.; Ferrari, P. F.; Gesierich, B.; Rozzi, S.; Chersi, F. y Rizzolatti, G. (2005). Parietal Lobe: From Action Organization to Intention Understanding. Science, 308(5722), 662-667.

Iacoboni, M.; Molnar-Szakacs, I.; Gallese, V.; Buccino, G.; Mazziotta, J.; y Rizzolatti, G. (2005). Grasping the intentions of others with one's owns mirror neuron system. PLoS Biology, 3(3), e79.

Leman, M. (2008). Embodied Music Cognition and Mediation Technology. Cambridge, MA: MIT Press.

Leman, M., Desmet, F., Styns F., van Noorden L. y Meolants D. (2009). Sharing musical expression through embodied listening: A case study rase on chinese guqin music. Music Perception: An Interdisciplinary Journal, Vol. 26, No. 3, 263-278.

Martínez, I. C. y Pereira Ghiena, A. (2013). Percepción musical y experiencia de las formas de la vitalidad. La experiencia musical sentida a partir de la intención comunicativa del intérprete. En F. Shifres, M. Jacquier, D. Gonnet, M. I. Burcet y R. Herrera (Eds). Actas de XI Encuentro de Ciencias Cognitivas de la Música. Nuestro Cuerpo en Nuestra Música. Buenos Aires: SACCoM, pp. 441-448. 
Naveda, L. y Leman, M. (2011). Hypotheses on the choreographic roots of the musical meter. A case study on afro-brazilian dance and music. En A. Pereira Ghiena, P. Jacquier, M. Valles y M. Martínez (Eds.). Musicalidad Humana. Actas de la X Reunión Anual de la Sociedad Argentina para las Ciencias Cognitivas de la Música. Buenos Aires. SACCoM, pp. 477-495.

Reybrouck, M. (2005). Body, mind and music: musical semantics between experiential cognition and cognitive economy. Trans. Transcultural Music Review, 9 (Artículo 16). Consultado el 30 de marzo de 2016.

Stern, D. N. (2010). Forms of Vitality: Exploring Dynamic Experiences in Psychology, the Arts, Psychotherapy, and Development. New York: Oxford University Press.

\section{Partitura - DVD}

Liszt, F. Années de pélerinage, deuxième années - Italie.[Música impresa]: para piano. Urtext Edition. Ernst Herttrich (eds.) Hans-Martin Theopold, fingering. München: G. Henle Verlag. HN 174. (1978).

Barenboim, D. (2011). Barenboim plays Liszt. (Metropolitan Munich, 1985). EuroArts: 2066658 DVD Video.

\section{Biografia de la autora}

\section{Juliette Epele}

epelejuliette@gmail.com

Juliette Epele es maestrando en Psicología de la Música de la Facultad de Bellas Artes de la Universidad Nacional de La Plata, donde se desempeña como ayudante de docencia en Lectura Pianística. Es, también, profesor de Piano y de Música de Cámara del Bachillerato especializado en artes de la misma universidad. Desde los inicios, integra el laboratorio para el estudio de la experiencia musical LEEM (FBA-UNLP) centrado en el estudio y desarrollo de la investigación científica en el campo de la cognición y la práctica musical. Su desarrollo académico se vincula a la ejecución e interpretación en el piano bajo una perspectiva corporeizada y situada de la expresión musical. Como músico, realizó estudios superiores de piano con la maestra Elsa Carranza en el Conservatorio Provincial "Gilardo Gilardi” de La Plata, y participó de cursos de perfeccionamiento dictados por los maestros Stephen Drury, Craig Ketter, Jordi Mora y Edith Fischer. 